\title{
Ground States in Classical Lattice Systems with Hard Core
}

\author{
R. SCHRADER \\ Seminar für theoretische Physik an der ETH (Zürich)
}

Received December 15, 1969

\begin{abstract}
In this paper we prove the existence of translation invariant ground states in an infinite classical lattice system with hard core and give a characterization of their support. Some examples are discussed.
\end{abstract}

\section{Introduction}

In the last years a great deal of effort has been spent on the investigation of equilibrium states of infinite systems in statistical mechanics, classical and quantum lattice systems, and continuous systems of particles with hard cores have been considered; their equilibrium states at temperature $T \neq 0$ have been investigated ${ }^{1}$. In a recent paper [2] Ruelle has started the investigation of the zero temperature states, i.e. the ground states, of the same systems. In this paper we shall study the zero temperature case in a classical lattice system with hard core.

\section{Definition of a Classical Lattice System with Hard Core}

Consider the lattice $\mathbb{Z}^{v}, v$ being a natural number and $\mathbb{Z}$ denoting the set of all integers. Usually the configuration space for a classical lattice system is taken to be $K=\{0,1\}^{\mathbb{Z}^{v}}$ which is compact, if we equip $\{0,1\}$ with the discrete topology (Tychonov's theorem). Each $\hat{X} \in K$ may be interpreted as the characteristic function of a unique set $X \cong \mathbb{Z}^{v}$. Conversely each $X \subseteq \mathbb{Z}^{v}$ defines a unique $\hat{X} \in K$, its characteristic function. Henceforth we will therefore identify the elements of $K$ with the subsets of $\mathbb{Z}^{v}$. If $x \in X$, we will say that the site $x$ is occupied in the configuration $X$. In a natural way $\mathbb{Z}^{v}$ acts as a transformation group $T$ on $K$ :

$$
T(a): X \rightarrow T(a) X=X+a, \quad X \cong \mathbb{Z}^{\nu}, a \in \mathbb{Z}^{\nu} .
$$

${ }^{1}$ For an account of this, see Ruelle [1] and the literature quoted therein. 
Now the Banachspace $\mathscr{B}$ of interactions is defined to consist of all real functions $\Phi$ on the finite subsets of $\mathbb{Z}^{v}$ such that $\Phi(\theta)=0$ and

$$
\begin{gathered}
\Phi(X+a)=\Phi(X) \text { for all } a \in \mathbb{Z}^{v} \text { and all } X \cong \mathbb{Z}^{v}(X \text { finite }) \\
\qquad \Phi \|:=\sum_{X \ni 0}|\Phi(X)|<\infty .
\end{gathered}
$$

Given $\Phi$, we associate to each finite $X \in K$ the interaction energy

Due to (2.1) we have

$$
U_{\Phi}(X):=\sum_{Y: Y \leqq X} \Phi(Y) .
$$

$$
U_{\Phi}(X+a)=U_{\Phi}(X) \text { for all } a \in \mathbb{Z}^{v} .
$$

Let $N(X)$ denote the number of points in $X$. Then

$$
\left|U_{\Phi}(X)\right| \leqq N(X)\|\Phi\| \text {. }
$$

The linear subspace

$$
\mathscr{B}_{0}:=\{\Phi \in \mathscr{B} \mid \exists X(\Phi) \text { finite, } \Phi(Y)=0 \text { for } 0 \in Y \nsubseteq X(\Phi)\}
$$

of $\mathscr{B}$ is called the space of finite range interactions. If $0 \neq \Phi_{0} \in \mathscr{B}_{0}$ we define its range to be

$$
\Delta_{0}=\bigcup_{X: \Phi_{0}(X) \neq 0, X \ni 0} X .
$$

Obviously $\Delta_{0}$ is finite and $0^{\circ} \in \Delta_{0}=-\Delta_{0}$ ([1], Page 16). From now on $\Phi_{0}$ will be arbitrary but fixed. We shall give a definition of a hard core determined by $\Phi_{0}$. This will amount to a specification of configurations which are allowed in the theory:

Definition 2.1. A configuration $X \in K$ is called allowed, if $\Phi_{0}(Y)=0$ for all finite $Y \leqq X$.

Note that $X$ is not supposed to be finite. Also the empty set is an allowed configuration. Denote by $K_{0}$ the set of all allowed configurations. Due to (2.1) and the definition we have the implications

$$
\begin{aligned}
X \in K_{0} & \Rightarrow X+a \in K_{0} \text { for all } a \in \mathbb{Z}^{v}, \\
Y \cong X \in K_{0} & \Rightarrow Y \in K_{0} .
\end{aligned}
$$

Furthermore we have the

Proposition 2.2. The set $K_{0}$ of all allowed configurations is compact.

Proof. Since $K$ is metrizable and compact, it is sufficient to prove sequential closedness. Now by definition $X_{l} \rightarrow X$ for $l \rightarrow \infty$ if and only if to each finite $\Lambda \subseteq \mathbb{Z}^{v}$ there exists $l(\Lambda)$ such that

$$
X_{l} \cap \Lambda=X \cap \Lambda \text { for } l \geqq l(\Lambda) \text {. }
$$


Suppose in particular that $X_{l} \in K_{0}$ for all $l$. We have to show that $X \in K_{0}$. Let $Y \leqq X$ be finite. Then we have

$$
X_{l} \supseteqq X_{l} \cap Y=Y \cap X=Y \text { for } l \geqq l(Y)
$$

and hence $\Phi_{0}(Y)=0$, because $X_{l} \in K_{0}$.

Since $Y \subseteq X$ was arbitrary we have $X \in K_{0}$, q.e.d.

The following remark will become important in the sequel: Let $\Lambda$ and $\Lambda^{\prime}$ be disjoint sets $\left(\Lambda \cap \Lambda^{\prime}\right)=0$ ) and let $X_{1}, X_{2} \in K_{0}, X_{1} \subseteq \Lambda, X_{2} \subseteq \Lambda^{\prime}$ be given. Then in general $X_{1} \cup X_{2} \notin K_{0}$ but we have

$$
\left.\begin{array}{c}
X_{1} \cup\left(\Lambda^{+} \cap X_{2}\right) \in K_{0} \\
\left(\Lambda^{+} \cap X_{1}\right) \cup X_{2} \in K_{0}
\end{array}\right\} \quad \text { if } \quad X_{1}, X_{2} \in K_{0}, X_{1} \subseteq \Lambda, X_{2} \subseteq \Lambda^{\prime}, \Lambda \cap \Lambda^{\prime}=0 .
$$

Here the map $\Lambda \rightarrow \Lambda^{+}$is defined as follows

$$
\Lambda^{+}:=\left\{x \in \Lambda \mid\left(\Delta_{0}+a\right) \cap \Lambda \neq \theta \text { and }\left(\Delta_{0}+a\right) \nsubseteq \Lambda \Rightarrow x \notin\left(\Delta_{0}+a\right)\right\} .
$$

Also we set

so that

$$
\Lambda^{-}:=\left\{x \in \Lambda \mid \exists a, x \in\left(\Delta_{0}+a\right) \nsubseteq \Lambda\right\}
$$

$$
\Lambda^{-}=\Lambda \backslash \Lambda^{+} \text {. }
$$

Roughly speaking $\Lambda^{+}$consists of all points in $\Lambda$ which have at least the distance $\Delta_{0}$ from the boundary of $\Lambda$. It is easy to prove that (2.8) holds.

\section{Infinite Volume Limit of the Ground State Energy}

The local minimum energy density for a given interaction $\Phi$ is obviously given by

for finite $\Lambda$, therefore

$$
E(\Phi, \Lambda):=\inf _{\substack{X: X \subseteq \Lambda \\ X \in K_{0}}} \frac{U_{\Phi}(X)}{N(\Lambda)}
$$

$$
\begin{aligned}
-\|\Phi\| \leqq E(\Phi, \Lambda) & \leqq 0 . \\
E(\Phi, \Lambda)+E(\Psi, \Lambda) & \leqq E(\Phi+\Psi, \Lambda) . \\
E(\Phi, \Lambda+a) & =E(\Phi, \Lambda) ; \quad a \in \mathbb{Z}^{v} .
\end{aligned}
$$

If we combine (3.1) and (3.2) we get

$$
|E(\Phi, \Lambda)-E(\Psi, \Lambda)| \leqq\|\Phi-\Psi\| .
$$

We want to prove the existence of the thermodynamic limit of $E(\Phi, \Lambda)$ if $\Lambda$ tends to infinity in the sense of van Hove ${ }^{2}$.

${ }^{2}$ We say that a sequence $\{\Lambda\}$ tends to infinity in the sense of van Hove (and we write $\Lambda \rightarrow \infty)$ if for every finite $X \leqq \mathbb{Z}^{v} N(\{x \mid x+X \leqq \Lambda\}) N^{-1}(\Lambda)$ tends to 1 . 
Let $\Phi$ be in $\mathscr{B}_{0}$ having range $\Delta$ and let $\Lambda \cap \Lambda^{\prime}=\theta$. Then we have $\left|N\left(\Lambda \cup \Lambda^{\prime}\right) E\left(\Phi, \Lambda \cup \Lambda^{\prime}\right)-N(\Lambda) E(\Phi, \Lambda)-N\left(\Lambda^{\prime}\right) E\left(\Phi, \Lambda^{\prime}\right)\right|$

$$
\begin{aligned}
& =\left|\inf _{\substack{X_{1} \subseteq A, X_{2} \subseteq A^{\prime} \\
X_{1} \cup X_{2} \in K_{0}}} U_{\Phi}\left(X_{1} \cup X_{2}\right)-\inf _{\substack{X_{1} \subseteq A ; X_{2} \subseteq \Lambda^{\prime} \\
X_{1}, X_{2} \in K_{0}}}\left\{U_{\Phi}\left(X_{1}\right)+U_{\Phi}\left(X_{2}\right)\right\}\right| \\
& \leqq \inf _{\substack{X_{1} \subseteq A, X_{2} \subseteq \Lambda^{\prime} \\
X_{1} \cup X_{2} \in K_{0}}} U_{\Phi}\left(X_{1} \cup X_{2}\right)-\inf _{\substack{X_{1} \subseteq A, X_{2} \subseteq A^{\prime} \\
X_{1} \cup X_{2} \in K_{0}}}\left\{U_{\Phi}\left(X_{1}\right)+U_{\Phi}\left(X_{2}\right)\right\} \mid \\
& +\left|\inf _{\substack{X_{1} \subseteq A, X_{2} \subseteq A^{\prime} \\
X_{1} \cup X_{2} \in K_{0}}}\left\{U_{\Phi}\left(X_{1}\right)+U_{\Phi}\left(X_{2}\right)\right\}-\inf _{\substack{X_{1} \subseteq \Lambda, X_{2} \subseteq A^{\prime} \\
X_{1}, X_{2} \in K_{0}}}\left\{U_{\Phi}\left(X_{1}\right)+U_{\Phi}\left(X_{2}\right)\right\}\right| .
\end{aligned}
$$

The first term may obviously be estimated by ([1], Prop. 2.3.1):

$$
\begin{aligned}
\sup _{X_{1} \leqq \Lambda, X_{2} \leqq \Lambda^{\prime}}\left|U_{\Phi}\left(X_{1} \cup X_{2}\right)-U_{\Phi}\left(X_{1}\right)-U_{\Phi}\left(X_{2}\right)\right| & \\
& \leqq \sup _{X_{1} \leqq \Lambda, X_{2} \subseteq \Lambda^{\prime}} N\left(X_{1}, X_{2}\right)\|\Phi\| \leqq N\left(\Lambda, \Lambda^{\prime}\right)\|\Phi\| .
\end{aligned}
$$

Here $N\left(X, X^{\prime}\right)$ is defined to be the number of points $a \in \mathbb{Z}^{v}$ such that

$$
X \cap(\Delta+a) \neq \theta \neq X^{\prime} \cap(\Delta+a) .
$$

$N\left(X, X^{\prime}\right)$ is obviously symmetric and monotone in both variables. We turn to the estimate of the second term.

Due to (2.7) we have

$$
\inf _{\substack{X_{1} \subseteq A, X_{2} \subseteq A^{\prime} \\ X_{1} \cup X_{2} \in K_{0}}}\left\{U_{\Phi}\left(X_{1}\right)+U_{\Phi}\left(X_{2}\right)\right\} \geqq \inf _{\substack{X_{1} \subseteq A, X_{2} \subseteq A^{\prime} \\ X_{1}, X_{2} \in K_{0}}}\left\{U_{\Phi}\left(X_{1}\right)+U_{\Phi}\left(X_{2}\right)\right\} .
$$

Now choose $\bar{X}_{1}, \bar{X}_{2}\left(\bar{X}_{1}, \bar{X}_{2} \in K_{0}, \bar{X}_{1} \subseteq \Lambda, \bar{X}_{2} \subseteq \Lambda^{\prime}\right)$ such that

$$
\inf _{\substack{X_{1} \subseteq A, X_{2} \subseteq A^{\prime} \\ X_{1}, X_{2} \in \bar{K}_{0}}}\left\{U_{\Phi}\left(X_{1}\right)+U_{\Phi}\left(X_{2}\right)\right\}=U_{\Phi}\left(\bar{X}_{1}\right)+U_{\Phi}\left(\bar{X}_{2}\right) .
$$

We have according to (2.8)

and hence

$$
\left(\bar{X}_{1} \cap \Lambda^{+}\right) \cup \bar{X}_{2} \in K_{0}, \quad \bar{X}_{1} \cup\left(\bar{X}_{2} \cap \Lambda^{\prime+}\right) \in K_{0}
$$

$$
\begin{aligned}
\inf _{\substack{X_{1} \subseteq A, X_{2} \subseteq \Lambda^{\prime} \\
X_{1} \cup X_{2} \in \bar{K}_{0}}}\left\{U_{\Phi}\left(X_{1}\right)+U_{\Phi}\left(X_{2}\right)\right\} & \inf _{\substack{X_{1} \subseteq A, X_{2} \subseteq \Lambda^{\prime} \\
X_{1}, X_{2} \in \bar{K}_{0}}}\left\{U_{\Phi}\left(X_{1}\right)+U_{\Phi}\left(X_{2}\right)\right\} \mid \\
& \leqq\left|U_{\Phi}\left(\bar{X}_{1} \cap \Lambda^{+}\right)-U_{\Phi}\left(\bar{X}_{1}\right)\right| \\
& \leqq\left|U_{\Phi}\left(\bar{X}_{1} \backslash \Lambda^{+}\right)\right|+N\left(\bar{X}_{1} \backslash \Lambda^{+}, \bar{X}_{1} \cap \Lambda^{+}\right)\|\Phi\| \\
& \leqq\left(N\left(\Lambda^{-}\right)+N\left(\Lambda^{-}, \Lambda^{+}\right)\right)\|\Phi\| .
\end{aligned}
$$

Analogously we get the estimate

Put

$$
\leqq\left(N\left(\Lambda^{\prime-}\right)+N\left(\Lambda^{\prime-}, \Lambda^{\prime+}\right)\right)\|\Phi\| .
$$

$\bar{N}\left(\Lambda, \Lambda^{\prime}\right):=N\left(\Lambda, \Lambda^{\prime}\right)+\operatorname{Min}\left(N\left(\Lambda^{-}\right)+N\left(\Lambda^{-}, \Lambda^{+}\right), N\left(\Lambda^{\prime-}\right)+N\left(\Lambda^{\prime-}, \Lambda^{\prime+}\right)\right)$. 
We have therefore proved the

Proposition 3.1. Let $\Phi$ have finite range $\left(\Phi \in \mathscr{B}_{0}\right)$ and let $\bar{N}\left(\Lambda, \Lambda^{\prime}\right)$ be defined by (3.5) with $\Lambda \cap \Lambda^{\prime}=0$. Then

$$
\begin{aligned}
& \left|N\left(\Lambda \cup \Lambda^{\prime}\right) E\left(\Phi, \Lambda \cup \Lambda^{\prime}\right)-N(\Lambda) E(\Phi, \Lambda)-N\left(\Lambda^{\prime}\right) E\left(\Phi, \Lambda^{\prime}\right)\right| \\
& \leqq \bar{N}\left(\Lambda, \Lambda^{\prime}\right)\|\Phi\| \text {. }
\end{aligned}
$$

Since $\bar{N}\left(\Lambda, \Lambda^{\prime}\right)$ has properties similar to $N\left(\Lambda, \Lambda^{\prime}\right)^{3}$, this gives, using standard arguments ([1], Theorem 2.4.1)

Theorem 3.2. If $\Phi \in \mathscr{B}$, the following limit exists

$$
E(\Phi)=\lim _{\Lambda \rightarrow \infty} E(\Phi, \Lambda)
$$

for any sequence $\{\Lambda\}$ which goes to infinity in the sense of van Hove. Furthermore

$(\alpha)-\|\Phi\| \leqq E(\Phi) \leqq 0 ; E(\lambda \Phi)=\lambda E(\Phi) ; \lambda \in \mathbb{R}^{+}$,

$(\beta)|E(\Phi)-E(\Psi)| \leqq\|\Phi-\Psi\|$,

$(\gamma)$ The function $E(\cdot)$ is concave on $\mathscr{B}: E(\Phi)+E(\Psi) \leqq E(\Phi+\Psi) \leqq 0$.

\section{Ground States}

Let $\mathscr{C}\left(K_{0}\right)$ be the Banach space of continuous functions on $K_{0}$. A probability measure $\varrho$ on $K_{0}$ is defined to be a linear form on $\mathscr{C}\left(K_{0}\right)$ such that

$$
\begin{aligned}
& \varrho(f) \geqq 0 \text { for } f \geqq 0 ; f \in \mathscr{C}\left(K_{0}\right) \\
& \varrho(\mathbb{I})=1 .
\end{aligned}
$$

Such a linear form is automatically continuous. For $f \in \mathscr{C}\left(K_{0}\right)$ set $f_{a}(X)=f(X-a)\left(a \in \mathbb{Z}^{v}\right)$ so that $f_{a} \in \mathscr{C}\left(K_{0}\right)$. Furthermore define the translate $\varrho_{a}$ of $\varrho$ by $\varrho_{a}(f)=\varrho\left(f_{a}\right)$. Evidently $\varrho_{a}$ is a probability measure if $\varrho$ is.

Definition 4.1. Given $\Phi \in \mathscr{B}$, a probability measure $\varrho$ on $K_{0}$ is defined to be a ground state, if for all sequences $\{\Lambda\}$ which tend to infinity in the sense of van Hove

$$
\lim _{\Lambda \rightarrow \infty} \sup _{a \in \mathbb{Z}^{2}} \frac{1}{N(\Lambda)} \varrho\left(U_{\Phi, \Lambda+a}\right)=E(\Phi)
$$

where $U_{\Phi, \Lambda}(X)=U_{\Phi}(X \cap \Lambda), \Lambda$ finite.

\footnotetext{
${ }^{3}$ Note that $\lim _{\Lambda \rightarrow \infty} N(\Lambda)^{-1} N\left(\Lambda^{-}\right)=O$, $\lim _{\Lambda \rightarrow \infty} N(\Lambda)^{-1} N\left(\Lambda^{-}, \Lambda^{+}\right)=O$
} 
Notice that $U_{\Phi, \Lambda} \in \mathscr{C}\left(K_{0}\right)$.

Obviously $\varrho_{a}\left(a \in \mathbb{Z}^{v}\right)$ is a ground state if $\varrho$ is one. More generally the convex hull $K(\varrho)$ of the translates of a ground state $\varrho$ consists of ground states. Finally we have the

Lemma 4.2. The weakly closed convex hull $\overline{K(\varrho)}$ of the translates of a ground state $\varrho$ consists of ground states.

Proof. Let $\bar{\varrho} \in \overline{K(\varrho)}$. Since

$$
E(\Phi, \Lambda)=E(\Phi, \Lambda+a) \leqq \frac{1}{N(\Lambda)} U_{\Phi, \Lambda+a}(X)
$$

for all $X \in K_{0}$ and all $a \in \mathbb{Z}^{v}$ and since $\varrho$ is a probability measure we have

$$
E(\Phi, \Lambda) \leqq \frac{1}{N(\Lambda)} \bar{\varrho}\left(U_{\Phi, \Lambda+a}\right) \quad\left(a \in \mathbb{Z}^{v}\right) .
$$

Therefore due to Theorem 3.2 we only have to show the following: Given $\varepsilon>0$ then for all sufficiently large $\Lambda$ (i.e. for $\Lambda \rightarrow \infty$ ) and all $a \in \mathbb{Z}^{v}$

$$
\frac{1}{N(\Lambda)} \bar{\varrho}\left(U_{\Phi, \Lambda+a}\right) \leqq E(\Phi)+\varepsilon .
$$

We know indeed that for all sufficiently large $\Lambda$ and all $a \in \mathbb{Z}^{v}$ we have

$$
\frac{1}{N(\Lambda)} \varrho\left(U_{\Phi, \Lambda+a}\right) \leqq E(\Phi)+\varepsilon .
$$

Therefore if $\varrho^{\prime}=\sum_{i} \lambda_{i} \cdot \varrho_{b_{i}} \in K(\varrho)$ we have

$$
\begin{aligned}
\frac{1}{N(\Lambda)} \varrho^{\prime}\left(U_{\Phi, \Lambda+a}\right) & =\sum_{i} \lambda_{i} \frac{1}{N(\Lambda)} \varrho\left(U_{\Phi, \Lambda+a+b_{i}}\right) \\
& \leqq E(\Phi)+\varepsilon .
\end{aligned}
$$

This yields (4.4) when $\varrho^{\prime} \rightarrow \bar{\varrho}$ weakly and Lemma 4.2 is proved.

Obviously the set $\overline{K(\varrho)}$ is invariant under translations, so once the existence of a ground state is established, Lemma 4.2 and a standard fixed point theorem (see e.g. [3], Page 645) show the existence of a translation invariant ground state.

We turn to the existence proof of a ground state and a characterization of translation invariant ground states. We will find that configurations belonging to the support of a translation invariant ground state are 
contained in a set which may be obtained from a local variational principle applied to a certain interaction energy density. More precisely define for finite $\Lambda \subseteq \mathbb{Z}^{v}$ and $X \in K_{0}$

$$
\begin{aligned}
G_{A}(X) & :=\sum_{\substack{Y: Y \subseteq X \\
Y \cap \Lambda \neq \theta}} \Phi(Y), \\
H_{\Lambda}(X) & :=\inf _{X^{\prime}: X^{\prime} \backslash \Lambda=X \backslash A} G_{\Lambda}\left(X^{\prime}\right)=\inf _{\substack{Y: Y \subseteq \Lambda \\
Y \cup(X \backslash A) \in K_{0}}} G_{\Lambda}(Y \cup(X \backslash \Lambda)) . \\
F_{\Lambda}(X) & :=G_{\Lambda}(X)-H_{\Lambda}(X) \geqq 0, \\
\mathscr{F}_{\Lambda} & :=\left\{X \in K_{0} \mid F_{\Lambda}(X)=0\right\} .
\end{aligned}
$$

We may write

where

$$
G_{\Lambda}(X)=\sum_{\substack{Y: Y \subseteq \mathbb{Z}^{v} \\ Y \cap A \neq \theta}} \Psi_{Y}(X)
$$

$$
\Psi_{Y}(X):=\left\{\begin{array}{lll}
\Phi(Y) & \text { if } & Y \cong X \\
0 & \text { if } & Y \nsubseteq X .
\end{array}\right.
$$

Since $\Psi_{Y} \in \mathscr{C}\left(K_{0}\right)$ and the r.h.s. of (4.9) is uniformly convergent (by (2.1) and (2.2)), we have $G_{A} \in \mathscr{C}\left(K_{0}\right)$. Indeed, if we write the norm on $\mathscr{C}\left(K_{0}\right)$ as

we have

$$
\||| f||=\sup _{X \in K_{0}}|f(X)|
$$

$$
\begin{aligned}
\left\|G_{\Lambda}\right\| & \leqq \sum_{\substack{Y: Y \subseteq \mathbb{Z}^{v} \\
Y \cap A \neq \theta}}\left\|\Psi_{Y}\right\|\left|\leqq \sum_{\substack{Y: Y \subseteq \mathbb{Z}^{v} \\
Y \cap \Lambda \neq \theta}}\right| \Phi(Y) \mid \\
& \leqq N(\Lambda)\|\Phi\|<\infty .
\end{aligned}
$$

Also $H_{A} \in \mathscr{C}\left(K_{0}\right)$. This is a consequence of the following remarks: If $X \rightarrow X_{0}$ then $Y \cup(X \backslash \Lambda) \rightarrow Y \cup\left(X_{0} \backslash \Lambda\right)$ for all $Y \leqq \Lambda$ and if $X$ is so close to $X_{0}$ that

$$
X \cap\left(\Lambda+\Delta_{0}\right)=X_{0} \cap\left(\Lambda+\Delta_{0}\right)
$$

then the conditions

$$
Y \cup(X \backslash \Lambda) \in K_{0} \quad \text { and } \quad Y \cup\left(X_{0} \backslash \Lambda\right) \in K_{0}
$$

are equivalent if $Y \cong \Lambda . H_{\Lambda}(X)$ is therefore continuous at $X_{0}$ as the lower envelope of a finite family of continuous functions at $X_{0}$.

Thus also $F_{A}=G_{A}-H_{A} \in \mathscr{C}\left(K_{0}\right)$ and $\mathscr{F}_{A}$ is a closed and hence compact set. 
Obviously $\mathscr{F}_{\Lambda}$ is not empty:

$L$ et $X \cong \Lambda, X \in K_{0}$ be such that $E(\Phi, \Lambda)=\frac{1}{N(\Lambda)} U_{\Phi}(X)$, then $X \in \mathscr{F}_{\Lambda}$. Remark now that if $\Lambda^{\prime} \supseteqq \Lambda$ and $X^{\prime} \backslash \Lambda=X \backslash \Lambda$

$$
\begin{aligned}
G_{\Lambda^{\prime}}(X)-G_{\Lambda^{\prime}}\left(X^{\prime}\right) & \\
= & \sum_{Y: Y \leqq X, Y \cap \Lambda^{\prime} \neq \theta} \Phi(Y)-\sum_{Y: Y \leqq X^{\prime}, Y \cap \Lambda^{\prime} \neq \theta} \Phi(Y) \\
& =\sum_{Y: Y \leqq X, Y \cap A \neq \theta} \Phi(Y)-\sum_{Y: Y \cong X^{\prime}, Y \cap \Lambda \neq \theta} \Phi(Y) \\
& =G_{\Lambda}(X)-G_{\Lambda}\left(X^{\prime}\right) .
\end{aligned}
$$

Since $X^{\prime} \backslash \Lambda=X \backslash \Lambda$ implies $X^{\prime} \backslash \Lambda^{\prime}=X \backslash \Lambda^{\prime}$ we immediately get

and hence

$$
F_{A^{\prime}}(X) \geqq F_{A}(X) \geqq 0
$$

This proves

$$
\mathscr{F}_{\Lambda^{\prime}} \cong \mathscr{F}_{\Lambda} \quad \text { for } \quad \Lambda^{\prime} \supseteqq \Lambda \text {. }
$$

Lemma 4.3. Let $\mathscr{F}_{\Lambda}$ be defined by (4.8). Then $\left\{\mathscr{F}_{\Lambda}\right\}_{\Lambda \text { finite } \leqq \mathbb{Z}^{v}}$ is a decreasingly filtered system of nonempty compact sets.

Theorem 4.4. The set $\mathscr{F}=\bigcap_{\Lambda \text { finite } \leqq \mathbb{Z}^{v}} \mathscr{F}_{\Lambda}$ is nonempty and compact. $\mathscr{F}$ is translation invariant.

Proof. The first part of the theorem is a consequence of Lemma 4.3 and a general theorem in topology (see e.g. [4]). The second part is a consequence of the following equalities

$$
G_{\Lambda}(X)=G_{\Lambda+a}(X+a) ; \quad H_{\Lambda}(X)=H_{\Lambda+a}(X+a) \quad \text { for all } a \in \mathbb{Z}^{\nu}
$$

since $\Phi(X+a)=\Phi(X)$. This gives

and therefore

$$
\mathscr{F}_{\Lambda+a}=\mathscr{F}_{\Lambda}+a
$$

$$
\mathscr{F}=\bigcap_{\Lambda \text { finite } \leqq \mathbb{Z}^{v}} \mathscr{F}_{\Lambda}=\bigcap_{\Lambda \text { finite } \leqq \mathbb{Z}^{v}} \mathscr{F}_{\Lambda+a}=\bigcap_{\Lambda \text { finite } \leqq \mathbb{Z}^{v}} \mathscr{F}_{\Lambda}+a=\mathscr{F}+a
$$

q.e.d.

Definition 4.5. A probability measure with support in $\mathscr{F}$ is called a strict ground state.

The following theorem shows that this definition is meaningful.

Theorem 4.6. Every strict ground state is a ground state.

Before we turn to the proof of this theorem let us first remark that

$$
U_{\Phi}(X \cap \Lambda)=G_{\Lambda}(X \cap \Lambda), \quad X \in K_{0} .
$$


Therefore due to the continuity of $G_{A}$ we expect the following chain of approximate equalities for $X \in \mathscr{F}$ :

$$
\begin{aligned}
\frac{1}{N(\Lambda)} U_{\Phi}(X \cap \Lambda) & =\frac{1}{N(\Lambda)} G_{\Lambda}(X \cap \Lambda) \approx \frac{1}{N(\Lambda)} G_{\Lambda}(X) \\
& =\frac{1}{N(\Lambda)} H_{\Lambda}(X) \approx \frac{1}{N(\Lambda)} H_{\Lambda}(X \cap \Lambda)=E(\Phi, \Lambda)
\end{aligned}
$$

which should hold for large $\Lambda$.

The following lemma will therefore be useful:

Lemma 4.7. Given $\varepsilon>0$ for all sufficiently large $\Lambda$

$$
\begin{gathered}
\frac{1}{N(\Lambda)}\left|G_{\Lambda}(X)-U_{\Phi}(X \cap \Lambda)\right| \leqq \frac{\varepsilon}{4}, \\
\left|\frac{1}{N(\Lambda)} H_{\Lambda}(X)-E(\Phi, \Lambda)\right| \leqq \frac{\varepsilon}{2}
\end{gathered}
$$

uniformly for all $X \in K_{0}$.

Proof of Lemma 4.7. Choose $\tilde{\Lambda}$ such that

$$
\sum_{\substack{W: W \ni 0 \\ W \neq \tilde{\Lambda}}}|\Phi(W)|<\frac{\varepsilon}{8} .
$$

Also for arbitrary finite sets $\Lambda, \Lambda^{\prime}$ define the following sets

$$
\begin{aligned}
& \Lambda^{-}\left(\Lambda^{\prime}\right):=\left\{x \in \Lambda \mid \exists a \in \mathbb{Z}^{v}, x \in\left(\Lambda^{\prime}+a\right) \nsubseteq \Lambda\right\} \\
& \Lambda^{+}\left(\Lambda^{\prime}\right):=\Lambda \backslash \Lambda^{-}\left(\Lambda^{\prime}\right) .
\end{aligned}
$$

Note that we have

$$
\Lambda^{+}=\Lambda^{+}\left(\Delta_{0}\right), \quad \Lambda^{-}=\Lambda^{-}\left(\Delta_{0}\right) .
$$

$\Lambda^{+}$and $\Lambda^{-}$where defined in Section 2.

Now

$$
\begin{aligned}
& \left|G_{\Lambda}(X)-U_{\Phi}(X \cap \Lambda)\right|=\left|\sum_{\substack{Y: Y \cap A \neq \theta, Y \subseteq X \\
Y \backslash \Lambda \neq \theta}} \Phi(Y)\right| \\
& \leqq \sum_{x \in \Lambda^{-}(\tilde{\Lambda})} \sum_{\substack{Y: Y \in x \\
Y \backslash \Lambda \neq \theta}}|\Phi(Y)|+\sum_{x \in \Lambda^{+}(\tilde{\Lambda})} \sum_{\substack{Y: Y \ni x \\
Y \backslash \Lambda \neq \theta}}|\Phi(Y)| .
\end{aligned}
$$

The first term may be estimated by

$$
N\left(\Lambda^{-}(\tilde{\Lambda})\right)\|\Phi\|
$$


and if $\Lambda$ is sufficiently large we have

$$
N\left(\Lambda^{-}(\tilde{\Lambda})\right) N^{-1}(\Lambda)<\frac{\varepsilon}{8\|\Phi\|} .
$$

In the second term by definition $Y$ is not contained in any translate of $\tilde{\Lambda}$. Therefore the second term may be estimated by $N\left(\Lambda^{+}(\tilde{\Lambda})\right) \frac{\varepsilon}{8}$ which is smaller than $N(\Lambda) \frac{\varepsilon}{8}$. Combining all estimates, (4.11) is proved.

To prove (4.12), suppose first

$$
\frac{1}{N(\Lambda)} H_{\Lambda}(X) \leqq E(\Phi, \Lambda)
$$

then due to the definitions and (4.11)

$$
\left|E(\Phi, \Lambda)-\frac{1}{N(\Lambda)} H_{\Lambda}(X)\right| \leqq \sup _{X \in K_{0}} \frac{1}{N(\Lambda)}\left|G_{\Lambda}(X)-U_{\Phi}(X \cap \Lambda)\right| \leqq \frac{\varepsilon}{4}
$$

for all sufficiently large $\Lambda$.

Next suppose

$$
E(\Phi, \Lambda) \leqq \frac{1}{N(\Lambda)} H_{\Lambda}(X)
$$

Choose $Y \leqq \Lambda, Y \in K_{0}$ such that $\frac{1}{N(\Lambda)} U_{\Phi}(Y)=E(\Phi, \Lambda)$. Since

$$
\begin{aligned}
\frac{1}{N(\Lambda)}\left|U_{\Phi}(Y)-U_{\Phi}\left(Y \cap \Lambda^{+}\right)\right| & =\frac{1}{N(\Lambda)}\left|\sum_{\substack{V: V \leqq Y \\
V \cap \Lambda^{-} \neq \theta}} \Phi(V)\right| \\
& \leqq \frac{N\left(\Lambda^{-}\right)}{N(\Lambda)}\|\Phi\| \leqq \frac{\varepsilon}{4}
\end{aligned}
$$

for all sufficiently large $\Lambda,(4.11)$ and (4.13) give

$$
\begin{aligned}
E(\Phi, \Lambda) & \leqq \frac{1}{N(\Lambda)} H_{\Lambda}(X) \leqq \frac{1}{N(\Lambda)} G_{\Lambda}\left(\left(Y \cap \Lambda^{+}\right) \cup(X \backslash \Lambda)\right) \\
& \leqq \frac{1}{N(\Lambda)} U_{\Phi}\left(Y \cap \Lambda^{+}\right)+\frac{\varepsilon}{4} \leqq E(\Phi, \Lambda)+\frac{\varepsilon}{2}
\end{aligned}
$$

proving (4.12) and hence Lemma 4.7.

Proof of Theorem 4.6. If $X \in \mathscr{F}$ we have $G_{\Lambda}(X)=H_{\Lambda}(X)$ for all $\Lambda$ and hence combination of (4.11) and (4.12) gives

$$
\left|\frac{1}{N(\Lambda)} U_{\Phi, \Lambda+a}(X)-E(\Phi, \Lambda)\right| \leqq \frac{3}{4} \varepsilon
$$


for all sufficiently large $\Lambda$ uniformly for all $X \in \mathscr{F}$ and $a \in \mathbb{Z}^{v}$. Here we used the translation invariance of $\mathscr{F}$. Choosing $\Lambda$ so large that

$$
|E(\Phi, \Lambda)-E(\Phi)| \leqq \frac{\varepsilon}{4}
$$

yields

$$
\left|\frac{1}{N(\Lambda)} U_{\Phi, \Lambda+a}(X)-E(\Phi)\right| \leqq \varepsilon .
$$

If $\varrho$ has support in $\mathscr{F}$ this gives in particular

$$
\left|\frac{1}{N(\Lambda)} \varrho\left(U_{\Phi, \Lambda+a}\right)-E(\Phi)\right| \leqq \varepsilon
$$

for all sufficiently large $\Lambda$ uniformly for all $a \in \mathbb{Z}^{\nu}$, i.e. $\varrho$ is a ground state and Theorem 4.6 is proved.

Conversely we have

Theorem 4.8. Every translation invariant ground state is a strict ground state.

Proof. First we assume $\Phi$ to have finite range $\Delta$ in order to make the essential argument more transparent. The extension of the proof to the general case is easy.

Define for any natural number $n$

so that

$$
\Lambda_{n}=\left\{x \in \mathbb{Z}^{v} \mid-n<x_{i} \leqq n ; i=1 \ldots v\right\}
$$

Since

$$
N\left(\Lambda_{n}\right)=(2 n)^{v} .
$$

we have

$$
\mathscr{F}=\left\{X \in K_{0} \mid F_{A_{n}}(X)=0 \text { for all } n \in \mathbb{Z}^{+}\right\}
$$

$$
K_{0} \mid \mathscr{F}=\bigcup_{n, m \in \mathbb{Z}^{+}}\left\{X \in K_{0} \mid F_{\Lambda_{n}}(X) \geqq \frac{1}{m}\right\} .
$$

Now assume that the translation invariant ground state $\varrho$ is not a strict ground state.

Then $\varrho\left(K_{0} \backslash \mathscr{F}\right)>0$ and there exist $p$ and $q$ such that

$$
\varrho(\mathscr{E})=\alpha>0 ; \quad \mathscr{E}=\left\{X \in K_{0} \mid F_{\Lambda_{p}}(X) \geqq \frac{1}{q}\right\} .
$$

Roughly speaking our aim is to change sufficiently many $X \in \operatorname{supp} \varrho$ in such a way that we obtain configurations $X^{\prime}$ which have a strictly lower energy density. Now choose $r \in \mathbb{Z}^{+}$so large that

$$
\Lambda_{r} \supseteqq \Lambda_{p}+\left(\Delta \cup \Delta_{0}\right)
$$


and let $T_{r}$ be the following subgroup of the translation group

$$
T_{r}=\left\{T(a) \in T \mid a=2 r \cdot \tau, \tau \in \mathbb{Z}^{\nu}\right\} .
$$

Put

$$
\begin{aligned}
& \Lambda_{r}(a)=T(a) \Lambda_{r}=\Lambda_{r}+a ; \quad T(a) \in T_{r}, \\
& \Lambda_{p}(a)=T(a) \Lambda_{p}=\Lambda_{p}+a ; \quad T(a) \in T_{r} .
\end{aligned}
$$

Then $\Lambda_{p}(a) \subseteq \Lambda_{r}(a)$ and $\left\{\Lambda_{r}(a)\right\}_{T(a) \in T_{r}}$ is a covering of $\mathbb{Z}^{v}$ by disjoint subsets.

Let $\chi_{\mathscr{E}}$ be the characteristic function of $\mathscr{E}$ and set

$$
\chi_{\mathscr{E}, a}(X)=\chi_{\mathscr{E}}(T(-a) X) ; \quad T(a) \in T_{r} .
$$

Consider $\chi_{\mathscr{\delta}, a}(X)$ for fixed $X \in K_{0}$ as a function of $a$. Assume $a_{j}(j=1 \ldots t)$ are such that $\chi_{\mathscr{E}, a_{j}}(X)=1$. Then by definition of $\mathscr{E}$ there exist

$$
Y_{X}^{j} \subseteq \Lambda_{p}\left(a_{j}\right) \subseteq \Lambda_{r}\left(a_{j}\right)
$$

such that $Y_{X}^{j} \cup\left(X \backslash \Lambda_{p}\left(a_{j}\right)\right) \in K_{0}$ and

In particular

$$
G_{A_{p}\left(a_{j}\right)}(X) \geqq \frac{1}{q}+G_{\Lambda_{p}\left(a_{j}\right)}\left(Y_{X}^{j} \cup\left(X \backslash \Lambda_{p}\left(q_{j}\right)\right) .\right.
$$

Moreover if we put

$$
G_{\Lambda_{p}\left(a_{j}\right)}(X) \geqq \frac{1}{q}+H_{\Lambda_{p}\left(a_{j}\right)}(X) .
$$

$$
\begin{aligned}
& \Lambda_{p}(\{a\})=\bigcup_{j=1}^{t} \Lambda_{p}\left(a_{j}\right) ; \quad \Lambda_{r}(\{a\})=\bigcup_{j=1}^{t} \Lambda_{r}\left(a_{j}\right), \\
& Y_{X}(\{a\})=\bigcup_{j=1}^{t} Y_{X}^{j} \cup\left(X \cap\left(\Lambda_{r}(\{a\}) \backslash \Lambda_{p}(\{a\})\right)\right)
\end{aligned}
$$

it is easy to see that

and that

$$
Y_{X}(\{a\}) \cup\left(X \backslash \Lambda_{r}(\{a\})\right) \in K_{0}
$$

$$
G_{\Lambda_{r}(\{a\})}(X) \geqq \frac{t}{q}+G_{\Lambda_{r}(\{a\})}\left(Y_{X}(\{a\}) \cup\left(X \backslash \Lambda_{r}(\{a\})\right)\right)
$$

More generally set

$$
\geqq \frac{t}{q}+H_{\Lambda_{r}(\{a\})}(X) \text {. }
$$

such that

$$
\Lambda_{r}^{n}=\bigcup_{a \in \Lambda_{n}} \Lambda_{r}(2 r \cdot a)
$$

$$
N\left(\Lambda_{r}^{n}\right)=N\left(\Lambda_{r}\right) N\left(\Lambda_{n}\right)=(2 r)^{v} N\left(\Lambda_{n}\right) .
$$


Then for all $X \in K_{0}$

$$
\begin{aligned}
\frac{1}{N\left(\Lambda_{r}^{n}\right)} G_{\Lambda_{r}^{n}}(X) \geqq & \frac{1}{q(2 r)^{v}} \cdot \frac{1}{N\left(\Lambda_{n}\right)} \cdot \sum_{a \in \Lambda_{n}} \chi_{\mathscr{E}, 2 r \cdot a}(X) \\
& +\frac{1}{N\left(\Lambda_{r}^{n}\right)} H_{\Lambda_{r}^{n}}(X) .
\end{aligned}
$$

Now due to (4.11) we have

$$
\frac{1}{N\left(\Lambda_{r}^{n}\right)}\left|\varrho\left(U_{\Phi, \Lambda_{r}^{n}}\right)-\varrho\left(G_{\Lambda_{r}^{n}}\right)\right| \leqq \frac{\varepsilon}{4}
$$

for all sufficiently large $n$. Since $\varrho$ is a ground state we therefore have

$$
\left|\frac{1}{N\left(\Lambda_{r}^{n}\right)} \varrho\left(G_{\Lambda_{r}^{n}}\right)-E\left(\Phi, \Lambda_{r}^{n}\right)\right| \leqq \frac{\varepsilon}{2} .
$$

Also (4.12) implies

$$
\left|\frac{1}{N\left(\Lambda_{r}^{n}\right)} \varrho\left(H_{\Lambda_{r}^{n}}\right)-E\left(\Phi, \Lambda_{r}^{n}\right)\right| \leqq \frac{\varepsilon}{2} .
$$

If we combine (4.14), (4.15) and (4.16) we get

$$
0 \leqq \varrho\left(\chi_{\mathscr{E}}^{n}\right) \leqq \varepsilon(2 r)^{v} \cdot q
$$

for $n \geqq n(\varepsilon)$. We have put

$$
\chi_{\varepsilon}^{n}=\frac{1}{N\left(\Lambda_{n}\right)} \sum_{a \in \Lambda_{n}} \chi_{\varepsilon, 2 r \cdot a} .
$$

Now we use the translation invariance of $\varrho$ which gives

$$
\varrho\left(\chi_{\mathscr{E}}^{n}\right)=\varrho\left(\chi_{\mathscr{E}}\right)=\alpha>0 .
$$

This, however, contradicts (4.17) if we choose $\varepsilon<\alpha(2 r)^{-v} \cdot q^{-1}$ and Theorem 4.8 is proved, if $\Phi \in \mathscr{B}_{0}$. Now let $\Phi \in \mathscr{B}$ be arbitrary.

With the notations as above choose $r$ so large that

(i) $\Lambda_{r} \supseteqq \Lambda_{p}+\Delta_{0}$

(ii) $\sum_{\substack{Y \cap \Lambda_{p} \neq \theta \\ Y \cap \mathbb{Z}^{v} \backslash \Lambda_{r} \neq \theta}}|\Phi(Y)|<c$,

where $c>0$ will be fixed in a moment. Since $\Phi \in \mathscr{B}$, it is always possible to satisfy these conditions. Repeating the above arguments it is easy to see that (4.14) is replaced by

$$
\begin{aligned}
\frac{1}{N\left(\Lambda_{r}^{n}\right)} G_{\Lambda_{r}^{n}}(X) \geqq & \frac{1}{(2 r)^{v} N\left(\Lambda_{n}\right)}\left\{\frac{1}{q}-4 c\right\} \sum_{a \in \Lambda_{n}} \chi_{\mathscr{E}, 2 r a}(X) \\
& +\frac{1}{N\left(\Lambda_{r}^{n}\right)} H_{\Lambda_{r}^{n}}(X) .
\end{aligned}
$$


Taking $c=(8 q)^{-1}$, we obtain (4.14) with $q$ replaced by $q^{\prime}=2 q$. Thus the arguments proceed as before.

Corollary 4.9. The translation invariant ground states form a weakly closed convex set.

\section{Examples}

In this section we want to discuss some examples which may be of interest. By definition $K_{0}$ depends on the hard core potential $\Phi_{0}$ and $\mathscr{F}$ depends in addition on $\Phi$. We have $\mathscr{F} \leqq K_{0} \leqq K$. $K_{0}=K$ if $\Phi_{0}=0$, $\mathscr{F}=K_{0}$ if $\Phi=0$. Let us denote by $\mathscr{G}$ the set of all invariant ground states. $\mathscr{G}$ is a weakly closed, nonempty convex set. We will be concerned with the following points:

a) The number of elements in $\mathscr{F}$.

$\beta)$ The number of linearly independent elements in $\mathscr{G}$.

$\gamma$ ) Existence of elements in $\mathscr{G}$ with nontrivial mean entropy.

The mean entropy of a translation invariant probability measure $\varrho$ is defined to be

$$
s(\varrho)=\lim _{\Lambda \rightarrow \infty}-\frac{1}{N(\Lambda)} \sum_{X \cong \Lambda} \varrho_{\Lambda}(X) \log \varrho_{\Lambda}(X),
$$

where $\left\{\varrho_{\Lambda}(\cdot)\right\}_{\Lambda \text { finite } \subseteq \mathbb{Z}^{v}}$ is the system of density distributions associated with $\varrho$ (see e.g. [1], Page 169). We always have $0 \leqq s(\varrho) \leqq \log 2$.

Now quite generally we may say the following. If $X \in \mathscr{F}$ and $X \neq \theta$, $X \neq \mathbb{Z}^{v}$ then $\mathscr{F}$ consists of at least two elements, for if $x \in X, y \notin X$ then $X \neq X^{\prime}=X+y-x \in \mathscr{F}$. The simplest case is of course $\Phi=\Phi_{0}=0$ such that $\mathscr{F}=K_{0}=K$. Clearly the number of linearly independent elements in $\mathscr{G}$ is infinite. Also there exist invariant ground states with nonzero mean entropy. Indeed

$$
\varrho_{\Lambda}^{(1)}(X)=\frac{1}{2^{N(\Lambda)}} ; \quad X \cong \Lambda
$$

defines a system of density distributions such that the corresponding $\varrho^{(1)} \in \mathscr{G}$ satisfies $s\left(\varrho^{(1)}\right)=\log 2$. If we put $\varrho^{(0)}(f)=f\left(\mathbb{Z}^{\nu}\right)$, then $\varrho^{(0)}$ is also in $\mathscr{G}$ and $s\left(\varrho^{(0)}\right)=0$. Since $s(\cdot)$ is affine on $\mathscr{G}([1]$, Page 183), the linear combination

$$
\varrho^{(\alpha)}=\alpha \varrho^{(1)}+(1-\alpha) \varrho^{(0)}, \quad 0 \leqq \alpha \leqq 1
$$

has mean entropy $s\left(\varrho^{(\alpha)}\right)=\alpha \log 2$, i.e. $s(\cdot)$ maps $\mathscr{G}$ onto the interval $[0, \log 2]$. Let us turn to more complicated examples. If $\Phi_{0}(\{x\})$ $=\lambda \neq 0\left(x \in \mathbb{Z}^{v}\right)$, then clearly $K_{0}$ consists only of the empty set, so we will assume $\lambda=0$ henceforth. Also we will assume $\Phi(X)=0$ if $N(X) \geqq 2$. Set $\Phi(\{x\})=\mu(=$ chemical potential $)$. If $\mu>0$ then $\mathscr{F}$ consists only of the 
empty set. If $\mu=0$, then $\mathscr{F}=K_{0}$ as remarked above. In case $\mu<0$ the problem of finding $\mathscr{F}$ will be called the problem of close packing. Now take

$$
\Phi_{0}(X)= \begin{cases}1 & \text { if } X=\{x, y\}, \quad x, y \text { nearest neighbours } \\ 0 & \text { else }\end{cases}
$$

Put

$$
\begin{aligned}
& X_{e}=\left\{x \in \mathbb{Z}^{v} \mid \sum_{i=1}^{v} x_{i} \text { even }\right\}, \\
& X_{0}=\left\{x \in \mathbb{Z}^{\nu} \mid \sum_{i=1}^{v} x_{i} \text { odd }\right\},
\end{aligned}
$$

so that

$$
\begin{aligned}
K_{0}=\mathscr{F}(\mu=0) & =\left\{X \subseteq \mathbb{Z}^{v} \mid X \subseteq X_{e} \text { or } X \cong X_{0}\right\} \\
\mathscr{F}(\mu<0) & =\left\{X_{e}, X_{0}\right\}
\end{aligned}
$$

For $\mu<0$ clearly $\mathscr{G}$ consists of only one element

$$
\varrho^{(2)}=\frac{1}{2} \delta_{X_{e}}+\frac{1}{2} \delta_{X_{0}}
$$

i.e.

$$
\varrho^{(2)}(f)=\frac{1}{2} f\left(X_{e}\right)+\frac{1}{2} f\left(X_{0}\right) .
$$

This is the ground state of the classical antiferromagnet and $s\left(\varrho^{(2)}\right)=0$. $\varrho^{(2)}$ also gives an example of spontaneous breakdown of symmetry ${ }^{4}$ : If we consider the subgroup $T_{e}$ of $T$ consisting of all even translations

$$
T_{e}=\left\{T(a) \in T \mid \sum_{i=1}^{v} a_{i} \text { even }\right\}
$$

then $\varrho^{(2)}$ decomposes into two extremal $T_{e}$-invariant probability measures, whereas $\varrho^{(2)}$ itself is an extremal $T$-invariant probability measure. If $\mu=0$ define $\varrho^{(3)}$ by

$$
\varrho_{\Lambda}^{3}(X)=\left\{\begin{array}{lll}
\frac{N\left(X_{e} \cap \Lambda\right)}{N(\Lambda)} \cdot \frac{1}{2^{N\left(X_{e} \cap \Lambda\right)}} & \text { if } & X \cong X_{e} \cap \Lambda \\
\frac{N\left(X_{0} \cap \Lambda\right)}{N(\Lambda)} \cdot \frac{1}{2^{N\left(X_{0} \cap \Lambda\right)}} & \text { if } & X \cong X_{0} \cap \Lambda \\
0 \quad \text { else } &
\end{array}\right.
$$

such that supp $\varrho^{(3)} \subset \mathscr{F}(\mu=0)$ and $s\left(\varrho^{(3)}\right)=\frac{1}{2} \log 2$. Again it is easy to see that $\mathscr{G}(\mu=0)$ is mapped onto $\left[0, \frac{1}{2} \log 2\right]$ by $s(\cdot)$.

\footnotetext{
${ }^{4}$ For an account of this cf. again Ruelle [1] and the literature quoted therein.
} 
More generally let

$$
\Phi_{0}(X)= \begin{cases}1 & \text { if } \quad X=\{x, y\}: \sum_{i=1}^{\tau}\left|x_{i}-y_{i}\right|=1 \\ 0 & \text { else }\end{cases}
$$

$(1 \leqq \tau \leqq v, \tau$ fixed).

For $\tau=v$ we have the example just discussed, so we will assume $\tau<v$. Then

$$
\begin{aligned}
& \mathscr{F}(\mu<0) \\
& =\left\{X \subseteq \mathbb{Z}^{v} \mid \exists g \in\{0,1\}^{\mathbb{Z}^{v-\tau}}: \sum_{i=1}^{\tau} x_{i} \equiv g\left(x_{\tau+1} \ldots x_{v}\right)(\bmod 2) \Leftrightarrow x \in X\right\}
\end{aligned}
$$

and

$$
\mathscr{F}(\mu=0)=K_{0}=\left\{X \cong \mathbb{Z}^{\nu} \mid \exists X^{\prime} \in \mathscr{F}(\mu<0): X \cong X^{\prime}\right\}
$$

so configurations belonging to $\mathscr{F}(\mu<0)$ show antiferromagnetic behaviour in direction of the first $\tau$ axes, whereas we have statistical behaviour in the remaining $v-\tau$ directions. Therefore $s(\varrho)=0$ for all $\varrho \in \mathscr{G}(\mu<0)$. Indeed it is easy to see that for any $\varrho \in \mathscr{G}(\mu<0)$

$$
N\left(\left\{X \subseteq \Lambda_{n} \mid \varrho_{A_{n}}(X)>0\right\}\right) \leqq 2^{(2 n)^{v-\tau}}
$$

since we have randomness only in $v-\tau$ directions. Also

for arbitrary

$$
-\sum_{i=1}^{t} a_{i} \log a_{i} \leqq \log \mathrm{t}
$$

so we get

$$
a_{i} \geqq 0 \quad \text { with } \quad \sum_{i=1}^{t} a_{i}=1
$$

$$
-\frac{1}{N\left(\Lambda_{n}\right)} \sum_{X \leqq A_{n}} \varrho_{A_{n}}(X) \log \varrho_{\Lambda_{n}}(X) \leqq \frac{(2 n)^{v-\tau}}{(2 n)^{v}} \log 2 .
$$

Letting $n$ tend to infinity proves the statement.

As a last example we will show that there exists a nontrivial hard core potential such that the corresponding problem of close packing gives invariant ground states with nonzero mean entropy. Indeed such an example is provided by the dimer problem: To fill a checker-board completely with dominos. This problem has been solved exactly [5]. Since the nonoverlap of the dominos becomes a hard-core condition, it is tempting to look whether this problem falls into the the general category of problems that have been considered here. With a slight modification this is indeed possible. We have to find a hard-core potential $\Phi_{0}$ which imitates the hard-core condition of the dimer problem. Of course we 
have $v=2 . \Phi_{0}$ is a combination of a two-body potential and a fourbody potential:

$$
\Phi_{0}(X)= \begin{cases}1 & \text { if } \quad X=\{x, y\}:\left|x_{1}-y_{1}\right|=\left|x_{2}-y_{2}\right|=1 \\ 1 & \text { if } \quad X=\left\{x^{1}, x^{2}, x^{3}, x^{4}\right\}: \frac{1}{2} \sum_{i=1}^{2} \sum_{j, k=1}^{4}\left|x_{i}^{j}-x_{i}^{k}\right|=10 \\ 0 & \text { else. }\end{cases}
$$

Then $\mathscr{F}(\mu<0)$ contains a translation invariant set $\tilde{\mathscr{F}}(\mu<0)$ where each configuration is a union of bits containing three sites linearly ordered. Locally each $X \in \tilde{\mathscr{F}}(\mu<0)$ is of the form

$$
\begin{aligned}
& \times \cdot \times \times \times \cdot \times \cdot \times \cdot \times \\
& \text {. . . . . } x \cdot x \cdot x \\
& \times \times \times \cdot \times \cdot x \cdot x \cdot x
\end{aligned}
$$

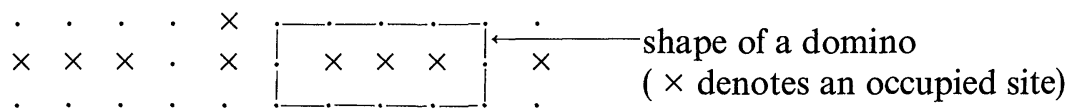

$$
\begin{aligned}
& \times \cdot \times \cdot \times \times \times \cdot \times \cdot \times \\
& x \cdot x \cdot \cdot \cdot \cdot \cdot x \cdot x \\
& x \cdot \times \cdot \times \times \times \cdot \times \times \times
\end{aligned}
$$

Since by definition combinations such as e.g.

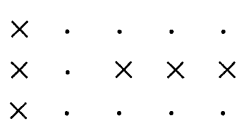

are not permitted for $X \in \tilde{\mathscr{F}}(\mu<0)$, it is easy to see that $\tilde{\mathscr{F}}(\mu<0)$ is properly contained in $\mathscr{F}(\mu<0)$. Apart from the translation group, which is larger in this formulation, $\tilde{\mathscr{F}}(\mu<0)$ just gives all solutions of the problem of close packing for the infinite dimer problem. Put

$$
\tilde{N}_{\Lambda}=\text { number of } X \cong \Lambda \text {, such that } X=Y \cap \Lambda \text { for some } Y \in \tilde{\mathscr{F}}(\mu<0) \text {. }
$$

Now for a rectangular $n \times m$ checker-board the number of ways of filling it with $\frac{1}{2} n \cdot m$ dimers is asymptotically equal to $\alpha^{\frac{1}{2} m n}$, with $\alpha=\exp \frac{2 G}{\pi}=1.791 \ldots(G=0.915 \ldots=$ Catalans number $)$. Therefore it is easy to see that for large

so if we set

$$
\tilde{N}_{\Lambda} \sim \alpha^{\frac{N(\Lambda)}{9}}
$$

$$
\varrho_{\Lambda}^{(4)}(X)=\left\{\begin{array}{l}
\frac{1}{\tilde{N}_{A}} \text { if } X=Y \cap \Lambda \text { for some } \quad Y \in \tilde{\mathscr{F}}(\mu<0) \\
0 \text { else }
\end{array}\right.
$$

18 Commun. math. Phys., Vol. 16 
we get $s\left(\varrho^{(4)}\right)=\frac{2}{9 \pi} G$. Again it is easy to see that $s(\cdot)$ maps the set of all invariant probability measures with support in $\tilde{\mathscr{F}}(\mu<0)$ onto the interval $\left[0, \frac{2}{9 \pi} G\right]$.

Acknowledgements. It is a great pleasure to thank Prof. D. Ruelle for proposing this problem and for suggesting various improvements in the proofs. A discussion with Prof. M. Fierz has been very helpful.

\section{Literature}

1. Ruelle, D.: Statistical mechanics. New York: Benjamin 1969.

2. - Commun. math. Phys. 11, 339 (1969).

3. Kantorovich, L. V., Akilov, G. P.: Functional analysis in normed spaces. New York: Pergamon 1964.

4. Bourbaki, N.: Topologie general. Paris: Hermann 1961.

5. Fisher, M. E.: Phys. Rev. 124, 1664 (1961).

Temperley, H. N. V.: Phil. Mag. 6, 1061 (1961).

Kasteleyn, P. W.: Physica 27, 1209 (1961).

Fisher, M. E., Stephenson, J.: Phys. Rev. 132, 1411 (1963).

R. Schrader

Physics Department

Harvard University

Cambridge, Mass. 02138, USA 